\section{ECONOMIC EVALUATION OF NEUROPSYCHIATRIC (NP) LUPUS IN AN INTERNATIONAL INCEPTION COHORT USING A MULTISTATE MODEL APPROACH}

${ }^{1}$ Ann E Clarke*, ${ }^{2}$ John G Hanly, ${ }^{3}$ Yvan St Pierre, ${ }^{4}$ Caroline Gordon, ${ }^{5}$ Sang-Cheol Bae, ${ }^{6} J u a n i t a$ Romero-Diaz, ${ }^{7}$ Jorge Sanchez-Guerrero, ${ }^{8}$ Sasha Bernatsky, ${ }^{9}$ Daniel J Wallace, ${ }^{10}$ David A Isenberg, ${ }^{10}$ Anisur Rahman, ${ }^{11}$ Joan T Merrill, ${ }^{12}$ Paul R Fortin, ${ }^{7}$ Dafna D Gladman, ${ }^{7}$ Murray B Urowitz, ${ }^{13}$ lan N Bruce, ${ }^{14}$ Michelle Petri, ${ }^{15}$ Ellen M Ginzler, ${ }^{16}$ Mary Anne Dooley, ${ }^{17}$ Rosalind Ramsey-Goldman, ${ }^{18}$ Susan Manzi, ${ }^{19}$ Andreas Jönsen, ${ }^{20}$ Graciela S Alarcón, ${ }^{21}$ Ronald FVan Vollenhoven, ${ }^{22}$ Cynthia Aranow, ${ }^{22}$ Meggan Mackay, ${ }^{23}$ Guillermo RuizIrastorza, ${ }^{24} \mathrm{~S}$ Sam Lim, ${ }^{25}$ Murat Inanc, ${ }^{26}$ Kenneth C Kalunian, ${ }^{27}$ Soren Jacobsen, ${ }^{28} \mathrm{Christine}$ A Peschken, ${ }^{29}$ Diane L Kamen, ${ }^{30}$ Anca Askanase, ${ }^{31}$ Vernon Farewell. ${ }^{1}$ University of Calgary, Alberta, Canada; ${ }^{2}$ Queen Elizabeth II Health Sciences Centre and Dalhousie University, Halifax, Nova Scotia, Canada; ${ }^{3}$ Research Institute of the McGill University Health Center, Montreal, Canada; ${ }^{4}$ Rheumatology Research Group, Institute of Inflammation and Ageing, College of Medical and Dental Sciences, University of Birmingham, Birmingham, UK; ${ }^{5}$ Hanyang University Hospital for Rheumatic Diseases, Seoul, Korea; 'Instituto Nacional de Ciencias Médicas y Nutricion, Mexico City, Mexico; ${ }^{7}$ Centre for Prognosis Studies in the Rheumatic Diseases, Toronto Western Hospital and University of Toronto, Toronto, Ontario, Canada; ${ }^{8}$ McGill University Health Centre, Montreal, Canada; ${ }^{9}$ Cedars-Sinai/David Geffen School of Medicine at the University of California, Los Angeles, California, USA; ${ }^{10}$ University College London, London, UK; ${ }^{11}$ Department of Clinical Pharmacology, OKlahoma Medical Research Foundation, Oklahoma City, OK, USA; ${ }^{12} \mathrm{CHU}$ de Québec -Universite Laval, Québec City, Canada; ${ }^{13}$ Arthritis Research UK Epidemiology Unit, Institute of Inflammation and Repair, Manchester Academic Health Sciences Centre, the University of Manchester, and NIHR Manchester Musculoskeletal Biomedical Research Unit, Central Manchester University Hospitals National Health Service Foundation Trust, Manchester Academic Health Science Centre, Manchester, UK; ${ }^{14}$ Johns Hopkins University School of Medicine, Baltimore, Maryland, USA; ${ }^{15}$ State University of New York Downstate Medical Center, Brooklyn, New York, USA; ${ }^{16}$ Thurston Arthritis Research Center, University of North Carolina, Chapel Hill, NC, USA; ${ }^{17}$ Northwestern University and Feinberg School of Medicine, Chicago, Illinois, USA; ${ }^{18}$ University of Pittsburgh School of Medicine, Pittsburgh, Pennsylvania, USA; ${ }^{19}$ Lund University, Lund, Sweden; ${ }^{20}$ University of Alabama at Birmingham, USA; ${ }^{21}$ University of Amsterdam, Rheumatology and Immunology Center, Amsterdam, Noord-Holland, NL; ${ }^{22}$ Feinstein Institute for Medical Research, Manhasset, New York, USA; ${ }^{23}$ BioCruces Health Research Institute, Hospital Universitario Cruces, University of the Basque Country, Barakaldo, Spain; ${ }^{24}$ Emory University School of Medicine, Atlanta, Georgia, USA; ${ }^{25}$ Istanbul University, Istanbul, Turkey; ${ }^{26}$ University of California Los Angeles School of Medicine, La
Jolla, California, USA; ${ }^{27}$ Copenhagen Lupus and Vasculitis Clinic, Rigshospitalet, Copenhagen University Hospital, Copenhagen, Denmark; ${ }^{28}$ University of Manitoba, Winnipeg, Manitoba, Canada; ${ }^{29}$ Medical University of South Carolina, Charleston, USA; ${ }^{30}$ Hospital for Joint Diseases, New York University Seligman Center for Advanced Therapeutics, New York, New York, USA; ${ }^{31}$ University of Cambridge, Cambridge, UK

\subsection{6/lupus-2021-lupus21century.67}

Background Little is known about the economic burden of NP lupus. We estimated direct and indirect costs (DC, IC) associated with NP events attributed to SLE and non-SLE causes using multistate modelling.

Methods Patients fulfilling ACR classification criteria for SLE from 31 centres in 11 countries were enrolled within 15 months of diagnosis. NP events were documented annually using ACR NP definitions and attributed to SLE or non-SLE causes. At each assessment and for SLE and non-SLE events, patients were stratified into 1 of 3 NP states (no, resolved, or new/ongoing NP event). The change in NP status characterized by transition rates between states was analyzed using multistate modelling (doi:10.1002/art.41876).

At each assessment, annual DC and IC were based on health resource use and lost work-force/non-work-force productivity over the preceding year. Resource use was costed using 2021 Canadian prices and lost productivity using Statistics Canada age-and-sex specific wages. Costs associated with SLE and non-SLE NP states were calculated by averaging all observations in each NP state. Multiple regressions adjusted for possible confounding of age at diagnosis, sex, race/ethnicity, disease duration, geographic region, education, and smoking on the association of annual DC and IC and NP state. 5 and 10-year cumulative costs for NP states were predicted by multiplying adjusted annual costs for each state by the expected state duration, forecasted using multistate modelling.

Abstract 1124 Table 1 Predicted Annual and 5 and 10-Yr Direct and Indirect Costs (in 2021 Canadian dollars) Stratified by (a) SLE and (b) Non-SLE NP States*

(a) SLE NP States

\begin{tabular}{|c|c|c|c|c|c|c|c|}
\hline & & \multirow[t]{2}{*}{ State $1^{* *}$} & \multirow[t]{2}{*}{ State 2} & \multirow[t]{2}{*}{ State 3} & Difference & Difference & Difference \\
\hline & & & & & State $1 \& 2$ & State $1 \& 3$ & State $2 \& 3$ \\
\hline \multirow[t]{3}{*}{ Direct Costs*** } & 1 year & 6715 & 9020 & 10809 & $2304(-2439,7048)$ & $4093(114,8072)$ & $1789(-3769,7346)$ \\
\hline & 5 years & 35567 & 45782 & 52060 & $10215(-12590,33020)$ & $16493(-4056,37041)$ & $6278(-20421,32976)$ \\
\hline & 10 years & 72307 & 91193 & 99496 & $18886(-25576,63348)$ & $27188(-14884,69261)$ & $8302(-43120,59725)$ \\
\hline \multirow[t]{3}{*}{ Indirect Costs $* * * *$} & 1 year & 24805 & 25133 & 34939 & $328(-5191,5846)$ & $10134(4310,15958)$ & $9806(3024,16589)$ \\
\hline & 5 years & 136970 & 138257 & 172674 & $1287(-25270,27844)$ & $35704(7705,63702)$ & $34417(2800,66033)$ \\
\hline & 10 years & 289343 & 293639 & 339156 & $4296(-48915,57506)$ & $49813(-5662,105287)$ & $45517(-15111,106145)$ \\
\hline
\end{tabular}

b) Non-SLE NP States

\begin{tabular}{|c|c|c|c|c|c|c|c|}
\hline & & State 1 & State 2 & State 3 & Difference & Difference & Difference \\
\hline & & & & & State $1 \& 2$ & State $1 \& 3$ & State $2 \& 3$ \\
\hline \multirow[t]{3}{*}{ Direct Costs $* * *$} & 1 year & 6401 & 8225 & 8868 & $1824(-1412,5059)$ & $2467(-676,5610)$ & $644(-3117,4404)$ \\
\hline & 5 years & 34587 & 41847 & 44002 & $7259(-9411,23930)$ & $9414(-6907,25736)$ & $2155(-16778,21088)$ \\
\hline & 10 years & 70890 & 83515 & 86262 & $12625(-22051,47301)$ & $15372(-18829,49574)$ & $2747(-35895,41390)$ \\
\hline \multirow[t]{3}{*}{ Indirect Costs**** } & 1 year & 22866 & 28195 & 34225 & $5330(466,10193)$ & $11359(6000,16717)$ & $6029(428,11630)$ \\
\hline & 5 years & 131194 & 151794 & 171979 & $20599(-3717,44915)$ & $40785(14979,66591)$ & $20186(-6291,46662)$ \\
\hline & 10 years & 279874 & 316701 & 342434 & $36827(-13437,87091)$ & $62560(10663,114457)$ & $25733(-26882,78348)$ \\
\hline
\end{tabular}

*Values are the mean $(95 \% \mathrm{Cl})$.

** State 1 = no NP event; State 2 = resolved NP event; State 3 = new/ongoing NP event.

*** Adjusted for age at diagnosis, race/ethnicity, and disease duration as the other predictors (sex, region, education, and smoking) were not significant.

**** Adjusted for sex, race/ethnicity, disease duration, region, education, and smoking as the other predictor (age at diagnosis) was not significant.

Boldface indicates differences which are significant as the $95 \% \mathrm{Cl}$ does not include 0 . 
Results 1697 patients (89\% female, 51\% non-Caucasian race/ ethnicity, mean age at enrolment 35.1 years) were followed a mean of 8.8 years. 1971 NP events occurred in 956 patients, $32 \%$ attributed to SLE. For SLE NP events, annual DC were higher in those with new/ongoing vs no events $(\$ 10,809$ vs \$6715) (table 1). Annual and 5-yr IC were higher in new/ ongoing vs no events and new/ongoing vs resolved events (5yr: new/ongoing vs no: $\$ 172,674$ vs $\$ 136,970)$. For non-SLE NP events, annual IC were higher in new/ongoing vs no events, new/ongoing vs resolved events, and resolved vs no events and 5 and 10-yr IC were higher in new/ongoing vs no events (10-yr: new/ongoing vs no: \$342,434 vs \$279,874). For all NP states, IC exceeded DC 2.8 to 4 -fold.

Conclusion IC are 1.3-fold higher in patients with new/ ongoing vs no NP events. While DC trended higher in new/ ongoing events, they were not significantly higher across all NP states and times. Impaired productivity associated with ongoing and resolved NP lupus is substantial, contributing to the previously documented reduced quality of life.

\section{$1200-$ COVID-19}

\section{FLARES AFTER SARS-COV-2 VACCINATION IN PATIENTS WITH SYSTEMIC LUPUS ERYTHEMATOSUS}

1,2,3 Medha Barbhaiya* ${ }^{4}$ Jonah M Levine, 1,2 Caroline Siegel, 1,2Vivian P Bykerk 1,2Deanna Jannat-Khah, 1,2,3 Lisa A Mandl. 'Division of Rheumatology; Hospital for Special Surgery, New York, NY, USA; ${ }^{2}$ Department of Medicine, Weill Cornell Medicine, New York, NY, USA; ${ }^{3}$ Department of Population Health Sciences, Weill Cornell Medicine, New York, NY, USA; ${ }^{4}$ Hospital for Special Surgery, New York, NY, USA

10.1136/lupus-2021-lupus21century.68

Background Vaccination against SARS-CoV-2 is particularly important for patients with systemic lupus erythematosus
(SLE), who may be at increased risk of hospitalization for COVID-19. However, the most common reason for vaccine refusal in patients with SLE is fear of SLE disease flare. Additionally, SARS-CoV-2 mRNA vaccines could potentially induce interferon production, associated with increased SLE disease activity. Thus far, no population-based data exist regarding whether SARS-CoV-2 vaccines trigger SLE flares.

Methods We e-mailed a survey on March 5, 2021 to 7,094 outpatients evaluated in our Rheumatology Division in New York City, to assess vaccination outcomes. ICD-10 algorithms identified patients with SLE. A self-reported disease flare was defined as 'a sudden worsening of your rheumatology condition or arthritis' within two weeks of the vaccine dose.

Results As of March 30, 2021, 2714 rheumatology patients responded (36.2\%). 136/466 (29.2\%) patients with SLE (mean [SD] age 54.7 [13.9] years; 93.4\% female; 67.7\% White; $13.2 \%$ Hispanic/Latinx) reported receiving at least one COVID-19 vaccine dose. Eighty-one patients (59.6\%) received Pfizer, 48 (39.3\%) received Moderna, and 4 (2.9\%) received Janssen. Of patients receiving Pfizer or Moderna, 72 (54.5\%) received $2 / 2$ doses. Twelve patients $(8.8 \%)$ reported SLE flare within two weeks of any COVID-19 vaccination (table 1). Patients reporting SLE flare were older (59.0 [14.0] versus 54.3 [13.9] years) and White (83.3\% versus 61.1\%). Flares occurred in $12.5 \%$ of patients receiving Moderna and $7.4 \%$ receiving Pfizer (6 patients each). Out of 7 patients receiving both vaccine doses and who reported a flare, 2 flared after both doses (table 1).

Of the 14 flares, 9 occurred after the first dose, and 5 occurred after the second dose. Most flares after the first vaccine dose were mild (77.8\%), whereas most after the second were moderate (60\%). 12/14 flares (85\%) were described as 'typical', predominantly characterized by joint pain, muscle aches, and fatigue. While 8/14 flares started 1 day after vaccination, 4/14 started 4-7 days later. Most SLE flares resolved

Abstract 1201 Table 1 Characteristics of Self-Reported Flares* After COVID-19 Vaccination Among Outpatients with Systemic Lupus Erythematosus

\begin{tabular}{|c|c|c|c|c|c|c|c|c|c|c|c|c|c|}
\hline \multirow[b]{2}{*}{$\begin{array}{l}\text { Study } \\
\text { ID }\end{array}$} & \multirow[b]{2}{*}{$\begin{array}{l}\text { Vaccine } \\
\text { Manufacturer }\end{array}$} & \multirow[b]{2}{*}{$\begin{array}{l}\text { Flare Onset } \\
\text { (Days after } \\
\text { vaccine dose) }\end{array}$} & \multirow[b]{2}{*}{$\begin{array}{l}\text { Flare Severity (Mild, } \\
\text { Moderate, Severe) }\end{array}$} & \multirow[b]{2}{*}{$\begin{array}{l}\text { "Typical" } \\
\text { Flare }\end{array}$} & \multirow[b]{2}{*}{$\begin{array}{l}\text { Flare } \\
\text { Duration } \\
\text { (in days) }\end{array}$} & \multirow[b]{2}{*}{ Fever } & \multicolumn{6}{|c|}{ Flare Symptoms** } & \multirow[b]{2}{*}{$\begin{array}{l}\text { Other } \\
\text { Symptoms }\end{array}$} \\
\hline & & & & & & & $\begin{array}{l}\text { Joint } \\
\text { pain }\end{array}$ & $\begin{array}{l}\text { Joint } \\
\text { swelling }\end{array}$ & $\begin{array}{l}\text { Skin } \\
\text { rash }\end{array}$ & Fatigue & $\begin{array}{l}\text { Muscle } \\
\text { aches }\end{array}$ & Other & \\
\hline \multicolumn{14}{|c|}{ Flare After Vaccine Dose \#1 } \\
\hline 1 & Moderna & 1 days & Mild & Yes & 1 days & 0 & 1 & 0 & 0 & 1 & 1 & 1 & Mouth sores \\
\hline 2 & Pfizer & 1 days & Moderate & Unknown & 4 days & 1 & 1 & 0 & 0 & 1 & 1 & 0 & \\
\hline 3 & Moderna & 1 days & Mild & Yes & 4 days & 0 & 1 & 1 & 0 & 0 & 0 & 0 & \\
\hline 4 & Pfizer & 1 days & Severe & Yes & 20 days & 0 & 1 & 0 & 0 & 1 & 0 & 1 & Brain fog \\
\hline 5 & Moderna & 1 days & Mild & Yes & 21 days & 0 & 1 & 0 & 1 & 1 & 1 & 0 & \\
\hline 6 & Moderna & 3 days & Mild & Yes & 22 days & 1 & 0 & 0 & 0 & 1 & 0 & 0 & \\
\hline 7 & Moderna & 4 days & Mild & Yes & 6 days & 0 & 0 & 0 & 0 & 1 & 1 & 0 & \\
\hline 8 & Pfizer & 4 days & Mild & Yes & 22 days & 1 & 0 & 0 & 0 & 1 & 1 & 0 & \\
\hline 9 & Pfizer & 7 days & Mild & Yes & 7 days & 0 & 0 & 0 & 1 & 0 & 0 & 0 & \\
\hline \multicolumn{14}{|c|}{ Flare After Vaccine Dose \#2 } \\
\hline 5 & Moderna & 1 day & Severe & No & 8 days & 0 & 1 & 1 & 0 & 1 & 1 & 0 & \\
\hline 6 & Moderna & 1 day & Mild & Yes & 4 days & 1 & 0 & 0 & 0 & 1 & 0 & 0 & \\
\hline 10 & Moderna & 1 day & Moderate & Yes & 3 days & 0 & 1 & 1 & 0 & 1 & 1 & 0 & \\
\hline 11 & Pfizer & 3 days & Moderate & Yes & 8 days & 0 & 1 & 1 & 0 & 1 & 1 & 1 & $\begin{array}{l}\text { Increased neuropathy, } \\
\text { neck pain, knee pain }\end{array}$ \\
\hline 12 & Pfizer & 7 days & Moderate & Yes & 2 days & 0 & 1 & 0 & 1 & 1 & 1 & 0 & \\
\hline
\end{tabular}

*12 patients reported 14 flares (2 patients flared at $2 / 2$ vaccine doses). 